\title{
Quadrupole and hexadecapole transition dipole moment alignment in fluorescent protein Homo-FRET
}

T. A. Masters, N. A. Robinson, R. J. Marsh, T. S. Blacker, D. A. Armoogum, et al.

T. A. Masters, N. A. Robinson, R. J. Marsh, T. S. Blacker, D. A. Armoogum, B. Larijani, A. J. Bain, "Quadrupole and hexadecapole transition dipole moment alignment in fluorescent protein Homo-FRET," Proc. SPIE 10672, Nanophotonics VII, 106720Y (4 May 2018); doi: 10.1117/12.2306401

SPIE. Event: SPIE Photonics Europe, 2018, Strasbourg, France 


\title{
Quadrupole and Hexadecapole Transition Dipole Moment Alignment in Fluorescent Protein Homo-FRET
}

\author{
T. A. Masters ${ }^{\mathrm{a}, \mathrm{b}}$, N. A. Robinson ${ }^{\mathrm{a}}$, R. J. Marsh a, d, T. S. Blacker ${ }^{\mathrm{a}, \mathrm{b}}$, D. A. \\ Armoogum $^{\mathrm{a}}$, B. Larijanic and A. J. Bain ${ }^{\mathrm{a}, \mathrm{b},{ }^{*}}$
}

(a) Department of Physics \& Astronomy, University College London, London, WC1E 6BT, UK (b) CoMPLEX, University College London, London, WC1E 6BT, UK (c) Cell Biophysics Laboratory, Ikerbasque, Basque Foundation for Science and Unidad de Biofisica (CSIC-UPV/EHU) Bilbao Spain (d) Current address: Randall Division of Cell and Molecular Biophysics, New Hunt's House, King's College London, Guy's Campus, London, SE1 1UL, UK.

*Author to whom correspondence should be addressed. Electronic mail: a.bain@ucl.ac.uk

\begin{abstract}
Polarized time resolved fluorescence measurements are used to characterise the structure of the two-photon tensor in the enhanced green fluorescent protein (EGFP) and predict the "hidden" degree of hexadecapole transition dipole alignment $\left\langle\alpha_{40}\right\rangle$ created by two-photon absorption (TPA). We employ a new method for the accurate STED measurement of the evolution of $\left\langle\alpha_{40}\right\rangle$ by analysing the saturation dynamics of the orthogonally polarized components of two-photon excited EGFP fluorescence as a function of the time delay between the $800 \mathrm{~nm}$ pump and $570 \mathrm{~nm}$ dump pulses. The relaxation of $\left\langle\alpha_{40}\right\rangle$ by homo-FRET is found to be considerably greater than that for the fluorescence anisotropy which directly measures the quadrupolar transition dipole moment alignment $\left\langle\alpha_{20}\right\rangle$. Our results indicate that higher order dipole moment correlation measurements promise to be a sensitive probe of resonance energy transfer dynamics.
\end{abstract}

Keywords: Two-photon, molecular alignment, FRET, rotational diffusion, Stimulated Emission Depletion, STED, fluorescent protein.

\section{INTRODUCTION}

In recent years, stimulated emission depletion (STED) from electronically excited states has found wide application as a means of achieving sub-wavelength image resolution in fluorescence microscopy ${ }^{1}$. STED, however, has its origins in high resolution spectroscopy with the ability to access high lying vibrational levels in molecular ground states that could not be probed by conventional absorption techniques ${ }^{2}$. STED has also been applied to time resolved spectroscopy ${ }^{3}$ and in the study of ultrafast vibrational relaxation dynamics within ground and excited electronic states ${ }^{4}$. In parallel to these developments, we have shown that polarized time-resolved STED can be used to circumvent single-photon electric dipole selection rules ${ }^{5}$ and allow the investigation of molecular orientation dynamics that are inaccessible to single photon spectroscopies ${ }^{6-11}$. Here we review the use of this latter property of STED to observe hitherto "hidden" hexadecapolar energy transfer dynamics arising from homo-FRET in two-photon excited enhanced green fluorescent protein (EGFP).

Nanophotonics VII, edited by David L. Andrews, Angus J. Bain, Jean-Michel Nunzi, Andreas Ostendorf, Proc. of SPIE Vol. 10672, 106720Y · @ 2018 SPIE · CCC code: 0277-786X/18/\$18 · doi: 10.1117/12.2306401 


\subsection{Orientational Distribution Function}

Short pulsed polarized laser excitation gives rise to an initial anisotropic distribution of molecular frame orientations in the laboratory frame of reference. In linearly polarized single- and multi-photon absorption, the excitation process has cylindrical symmetry about the polarization vector of the light ${ }^{12-14}$ which defines the laboratory frame Z-axis. Emission from the excited population is characterised by a rearrangement of electronic charge in the molecular frame giving rise to an emission transition dipole moment. The probability of finding an emission transition dipole moment oriented between the polar angles $\boldsymbol{\theta}$ and $\boldsymbol{\theta}+\boldsymbol{\delta} \boldsymbol{\theta}, \boldsymbol{\phi}$ and $\phi+\delta \phi$ can be expressed in terms of a spherical harmonic expansion ${ }^{15-17}$

$$
P_{E X}(\theta, \phi, t=0)=\sum_{K Q}\left\langle C_{K Q}(0)\right\rangle Y_{K Q}(\theta, \phi)
$$

The expansion coefficients $\left\langle\boldsymbol{C}_{\mathbf{K Q}}(\mathbf{0})\right\rangle$ define the moments of the distribution. As the excitation process has cylindrical symmetry about $\mathrm{Z}$ with an isotropic ground state population, this symmetry is transferred to the excited state distribution function and only even rank moments with $\boldsymbol{Q}=\mathbf{0}$ terms are allowed. As a result, the expansion can be reduced to an expansion in terms of Legendre polynomials of rank $\boldsymbol{K}$,

$$
P_{E X}(x, t=0)=\frac{1}{2}+\sum_{K>0} \frac{\left(1+(-1)^{K}\right)}{4} \sqrt{2 K+1}\left\langle\alpha_{K 0}(0)\right\rangle P_{K}(x), \quad x=\cos \theta
$$

where $\left\langle\alpha_{\mathrm{K} 0}(\mathbf{0})\right\rangle$ are the normalized moments of the distribution

$$
\left\langle\alpha_{K 0}(0)\right\rangle=\left\langle C_{K 0}(0)\right\rangle /\left\langle C_{00}(0)\right\rangle
$$

The excited state (transition dipole) population distribution is given by

$$
N_{E X}(x, t)=N_{E X}(t) P_{E X}(x, t)
$$

where $N_{E X}(t)$ is the total excited state population present at time $t$. For single-photon excitation from an isotropic ground state distribution, the expansion terminates at $\boldsymbol{K}=2$. With two-photon excitation, the expansion contains both quadrupolar $\left(\boldsymbol{K}=2,\left\langle\boldsymbol{\alpha}_{\mathbf{2} 0}\right\rangle\right)$ and hexadecapolar $\left(\boldsymbol{K}=4,\left\langle\boldsymbol{\alpha}_{40}\right\rangle\right)$ degrees of transition dipole alignment. The fluorescence anisotropy from the excited population is directly proportional to $\left\langle\alpha_{20}\right\rangle^{15}$,

$$
R(t)=\left\langle\alpha_{20}(t)\right\rangle / \sqrt{5}
$$

Electric dipole selection rules do not allow the contribution of moments greater than $\boldsymbol{K}=2$ in spontaneous emission ${ }^{15,16,18}$ and the direct observation of $\left\langle\alpha_{40}\right\rangle$ is not possible. For isotropic solutions, in the case of small step single axis symmetric rotational diffusion with diffusion coefficient $\boldsymbol{D}$, the moments of $\boldsymbol{P}_{\boldsymbol{E} X}(\boldsymbol{x}, \boldsymbol{t})$ evolve according to ${ }^{15,19,20}$,

$$
\left\langle\alpha_{K Q}(t)\right\rangle=\left\langle\alpha_{K Q}(0)\right\rangle \exp [-D K(K+1) t]
$$


The time dependent orientational distribution function for an even ordered array of transition dipoles is therefore,

$$
P_{E X}(x, t)=\frac{1}{2}+\sum_{K>0}\left(1+(-1)^{K}\right) \frac{\sqrt{2 K+1}}{4}\left\langle\alpha_{K 0}(0)\right\rangle \exp \left(\frac{-t}{\tau_{K 0}}\right) P_{K}(x)
$$

where,

$$
\tau_{20}=1 / 6 D, \quad \tau_{40}=1 / 20 D=0.3 \tau_{20}, \cdots \tau_{K 0}=6 \tau_{20} / K(K+1)
$$

Knowledge of one correlation function should provide complete information on the evolution of all other moments of the distribution. This relationship had not been tested experimentally until the development and application of six wave mixing techniques by Charra et al. ${ }^{21}$ and Meech and co-workers ${ }^{22}$ in which non-centrosymmetric order, characterized by $\boldsymbol{K}=1$ (dipolar alignment or orientation) and $\boldsymbol{K}=3$ (octupolar alignment), is created and probed by six wave nonlinear optical mixing with pump and probe fields at $\omega$ and $2 \omega$. If molecular motion is no longer diffusive (e.g. co-operative), Equation (8) no longer applies and the time evolution of moments in addition to $\left\langle\boldsymbol{\alpha}_{20}\right\rangle$ will provide new information and test dynamical theories ${ }^{23}$.

\subsection{Depolarization Dynamics and Resonance Energy Transfer}

Fluorescence depolarization can also result following resonance energy transfer ${ }^{24-26}$. In the case of transfer between identical molecules (homo-FRET), migration of the initial excitation to surrounding molecules leads to a partial (timedependent) randomization of the emission dipole moment alignment in the laboratory frame. Homo-FRET fluorescence depolarization has been investigated in Langmuir-Blodgett films ${ }^{27}$ and between rigidly held ${ }^{28}$ and rotationally diffusing $^{29,30}$ molecules in isotropic fluid media. To date we are unaware of any measurement or theoretical modeling of FRET depolarization (homo- or hetero-transfer) other than $\left\langle\alpha_{20}\right\rangle$. Given the intrinsic and greater sensitivity of $\left\langle\alpha_{40}\right\rangle$ to small step angular averaging as compared to $\left\langle\alpha_{20}\right\rangle$ (see Equation (8)), it is reasonable to expect that the degree of hexadecapolar depolarization due to energy migration will be more pronounced, affording the possibility of detecting and potentially quantifying energy transfer dynamics at lower concentrations (greater intermolecular distances) than permitted by conventional fluorescence anisotropy measurements. Theoretical treatments of time dependent fluorescence depolarization due to resonance energy transfer (homo-FRET) in the presence of molecular rotation have been advanced by a number of groups ${ }^{29,31}$. For EGFP, where the intrinsic rate of rotational depolarization is slow, the fluorescence anisotropy has been successfully modelled assuming independent molecular reorientation and FRET depolarization ${ }^{30}$,

$$
R(t)=R(0) \exp \left(-t / \tau_{20}\right) \exp \left(-\left(C R_{0}^{3} / 375\right)\left(t / \tau_{F}\right)^{1 / 2}\right)
$$

where $\boldsymbol{C}$ is the concentration (in $\mathrm{mM}$ ) of EGFP and $\boldsymbol{R}_{\boldsymbol{0}}$ is the Förster radius (in nm) for homo-transfer. From spectroscopic measurements a value for $\boldsymbol{R}_{\mathbf{0}}$ for homo-transfer in EGFP of $4.65 \mathrm{~nm}$ has been reported ${ }^{32}$, however fits to $\boldsymbol{R}(\boldsymbol{t})$ data using Equation (9) yielded a significantly larger value of $7.3( \pm 0.1) \mathrm{nm}^{30}$. In order to accurately determine the structure of the two-photon transition tensor in EGFP, both time resolved linear and circularly polarized anisotropy decays are needed ${ }^{33}$. These were recorded using time correlated single photon counting ${ }^{33-35}$ at a concentration of $1 \mu \mathrm{M}$ 
(see Figure 1(a)) where, from Equation (9), the contribution from homo-FRET to the EGFP anisotropy decay is seen to be negligible $\left(3 \times 10^{-3} \% \mathrm{~ns}^{-1}\right.$ for $\boldsymbol{R}_{\mathbf{0}}=4.65$ and $1.3 \times 10^{-2} \% \mathrm{~ns}^{-1}$ for $\left.\boldsymbol{R}_{\boldsymbol{0}}=7.3 \mathrm{~nm}\right)$.
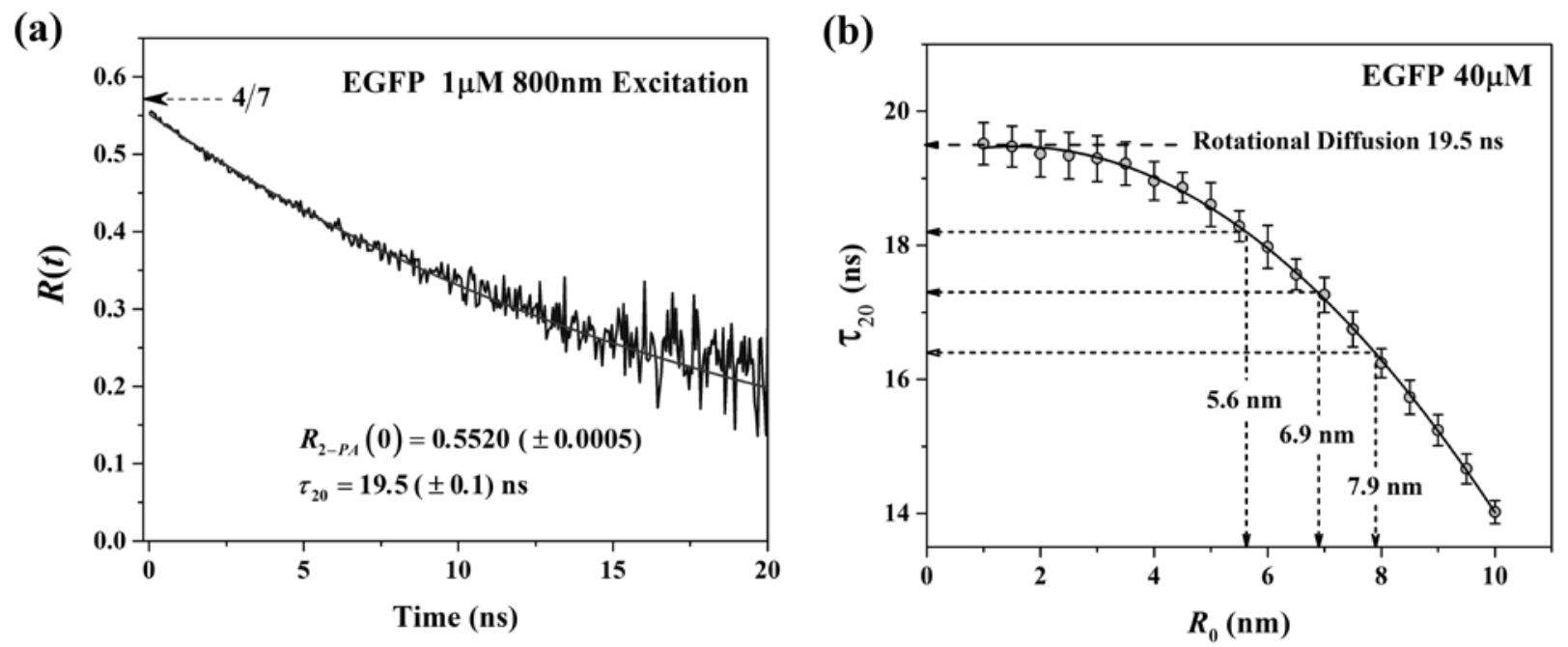

Figure 1(a). The fluorescence anisotropy decay of a $1 \mu \mathrm{M}$ solution of EGFP in phosphate-buffered saline (PBS) following linearly polarized two-photon excitation at $800 \mathrm{~nm}$. (b) Fits to simulated data using Equation (9) to observe the effect of homo-FRET on the anisotropy decay time.

In our laboratory, time resolved STED measurements made using a streak camera system require EGFP concentrations in the region of $40 \mu \mathrm{M}^{33}$. At these concentrations, the measured fluorescence anisotropy decay, whilst yielding a comparable initial anisotropy to that at $1 \mu \mathrm{M}(0.54 \pm 0.01)$ had a shorter correlation time of $17.3( \pm 0.9) \mathrm{ns}$. The faster decay indicates the presence of homo-FRET depolarization but was still well fitted by a single correlation time. To investigate this further, we simulated fluorescence anisotropy decays in MATLAB (The Mathworks) ${ }^{11}$, based on Equation (9), for a $40 \mu \mathrm{M}$ solution of EGFP using the average fluorescence lifetime of $2.75 \mathrm{~ns}^{34}$ an intrinsic rotational correlation time of $19.5 \mathrm{~ns}$ and an initial fluorescence anisotropy of 0.552 , taking values of $\mathrm{R}_{0}$ from $1 \mathrm{~nm}$ to $10 \mathrm{~nm}$. All decays were well fitted by a single exponential. A second order polynomial fit to the data points (see Figure 1b) indicates that the $40 \mu \mathrm{M}$ correlation time of $17.3( \pm 0.9)$ ns we observe corresponds to $\boldsymbol{R}_{\mathbf{0}} \approx 7( \pm 1) \mathrm{nm}$. Homo-FRET is thus an active depolarization mechanism for the quadrupolar transition dipole moment alignment and, in addition to molecular rotation, can also be expected to influence the relaxation of the hexadecapolar alignment prepared by two-photon excitation.

\section{TWO-PHOTON EXCITED STED}

A schematic Jablonski diagram illustrating the principles of STED following TPA in EGFP is shown in Figure 2(a). TPA at $800 \mathrm{~nm}$ of a $200 \mathrm{fs}$ laser pulse (pump) is followed by rapid radiationless relaxation ( $\mathbf{t}_{\mathbf{I C}}$ ) to lower vibrational levels of the $S_{1}$ singlet state. Application of a time delayed $(\Delta t)$ dump pulse leads to the preferential photo-de-selection of 
molecules whose transition dipole moments are closely aligned to the dump polarization. As a result there is an abrupt change in the fluorescence anisotropy in addition to the polarized and total fluorescence intensities. The efficiency of STED is, in addition to the STED cross section and orientational factors, dependent on the depopulation rate $\left(1 / t_{\text {VIB }}\right)$ of the terminating vibrational levels in $\mathrm{S}_{0}$ compared to the duration of the dump pulse $\left(\boldsymbol{t}_{\boldsymbol{D}}\right)$. If $\boldsymbol{t}_{\boldsymbol{D}}$ is shorter or comparable to $\boldsymbol{t} \boldsymbol{V} \boldsymbol{B}$, re-pumping of $\mathrm{S}_{1}$ becomes more marked with increasing dump energy and the degrees of population depletion and fluorescence depolarization are seen to saturate ${ }^{5,8}$. Typical results for EGFP are shown in Figure 3.

(a)

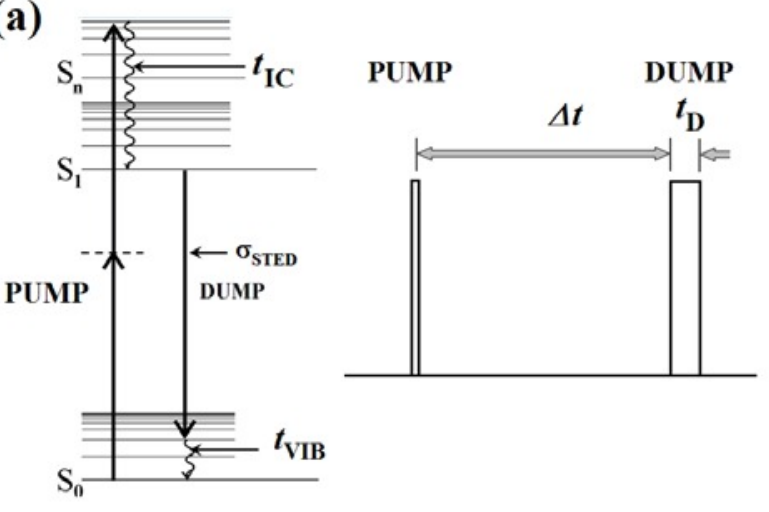

(b)

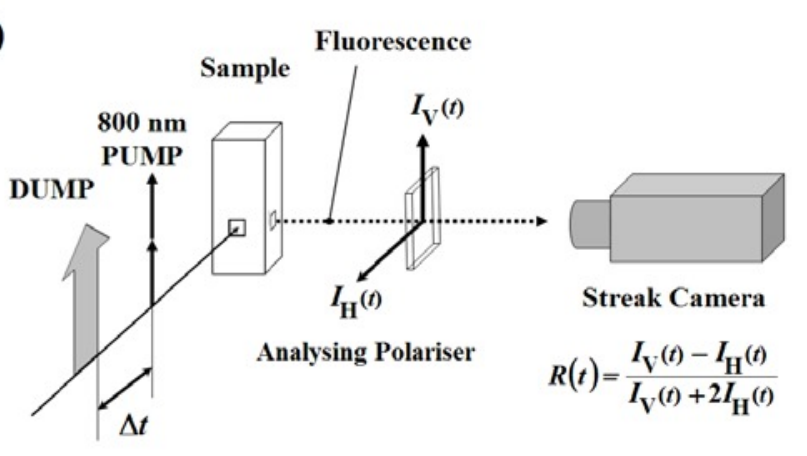

Figure 2: (a) Spontaneous and STED induced photophysical pathways following near infrared TPA (pump pulse). Following linearly polarized TPA there is rapid relaxation ( $\mathbf{t}_{\mathbf{I C}}$ sub-ps) to low lying vibrational levels of $\mathrm{S}_{1}$. At an experimentally variable time $\boldsymbol{\Delta t}$ a linearly polarized dump pulse is applied which removes population from $\mathrm{S}_{1}$. (b) The time resolved intensities for fluorescence polarized parallel $(\mathrm{V})$ and perpendicular $(\mathrm{H})$ to the excitation pulse are recorded by imaging the depletion region onto the entrance slit of a streak camera. From: T. A. Masters, N. Robinson, R. J. Marsh, D. A. Armoogum, T. S Blacker B. Larijani, and A. J. Bain. J. Chem. Phys. 148, 134312 (2018): used in accordance with the Creative Commons Attribution (CC BY) license.

\subsection{STED Depletion Theory}

In our experiments, the picosecond timescale of STED is significantly shorter than both the molecular reorientation and fluorescence lifetimes of EGFP and these contributions to the depletion dynamics can be neglected. With the sole approximation of a constant dump intensity over a time duration $\boldsymbol{t}_{\boldsymbol{D}}$, the rate equations for induced transitions between the excited state and the (initially unpopulated) upper vibrational levels of the ground state (see Figure 2(a)) immediately following the application of the dump pulse $t=\Delta t+t_{D}$ is given by,

$$
\begin{aligned}
& N_{E X}\left(x, \Delta t+t_{D}\right)=N_{E X}(\Delta t) P_{E X}(x, \Delta t) \times G_{D U M P} \\
& G_{D U M P}=\left[\frac{\exp \left(-\left(t_{D} / t_{V I B}+2 S x^{2}+d\right) / 2\right)}{2 d}\right]\left[\frac{t_{D}}{t_{V I B}}(\exp (d)-1)+d(\exp (d)+1)\right] \\
& d=\sqrt{\left(t_{D} / t_{V I B}\right)^{2}+4 S^{2} x^{4}}
\end{aligned}
$$


The STED observables are thus,

$$
\begin{aligned}
& F_{D}=1-\left[\int_{-1}^{1} G_{D U M P} N_{E X}(x, \Delta t) d x / \int_{-1}^{1} N_{E X}(x, \Delta t) d x\right] \\
& F_{D}^{V}=1-\left[\int_{-1}^{1} G_{D U M P} N_{E X}(x, \Delta t) x^{2} d x / \int_{-1}^{1} N_{E X}(x, \Delta t) x^{2} d x\right] \\
& F_{D}^{H}=1-\left[\int_{-1}^{1} G_{D U M P} N_{E X}(x, \Delta t)\left(1-x^{2}\right) d x / \int_{-1}^{1} N_{E X}(x, \Delta t)\left(1-x^{2}\right) d x\right] \\
& \Delta R=R(\Delta t)-\frac{1}{2}\left[\int_{-1}^{1} G_{D U M P} N_{E X}(x, \Delta t)\left(3 x^{2}-1\right) d x / \int_{-1}^{1} G_{D U M P} N_{E X}(x, \Delta t) d x\right]
\end{aligned}
$$

The integrals in Equation (13) were calculated using Mathematica (Wolfram Research). Figure 3 shows simulations of these as a function of $\boldsymbol{S}$ for values of $\boldsymbol{t}_{\boldsymbol{D}} / \boldsymbol{t}_{V I B}$ ranging from 50 and 0.02 for a two-photon excited population with an initial anisotropy and hexadecapolar alignment of 4/7 and 8/21 respectively. Population depletion and depolarization are both maximized with increasing $\boldsymbol{t}_{\boldsymbol{D}} / \boldsymbol{t}_{V I \boldsymbol{B}} ; \boldsymbol{F}_{\boldsymbol{D}}, \boldsymbol{F}_{\boldsymbol{D}}^{\boldsymbol{V}}$ and $\boldsymbol{F}_{\boldsymbol{D}}^{\boldsymbol{H}}$ are seen to saturate in the region where $\boldsymbol{S}>6$ with close to $100 \%$ depletion achieved when $\boldsymbol{t}_{\boldsymbol{D}} / \boldsymbol{t}_{\boldsymbol{V}} \boldsymbol{I B} \approx 50$. The maximum degree of population depletion attainable decreases as $\boldsymbol{t}_{\boldsymbol{D}} / \boldsymbol{t}_{V \boldsymbol{}} \boldsymbol{B}$ is reduced. When $\boldsymbol{t}_{\boldsymbol{D}}$ and $\boldsymbol{t}_{\boldsymbol{V} I \boldsymbol{B}}$ are equal this is approximately 0.68 . When $\boldsymbol{t}_{V I \boldsymbol{B}}>>\boldsymbol{t}_{\boldsymbol{D}}$, the population removed as the dump pulse is applied becomes bottlenecked in the upper ground state vibrational levels and the maximum degree of population depletion that is attainable tends to 0.5 . This corresponds to the limit observed in the continuous wave optical pumping of a two-level system in the absence of spontaneous emission ${ }^{36}$. The change in fluorescence anisotropy shows a high degree of sensitivity to the degree of excited state re-pumping. When $\boldsymbol{t}_{\boldsymbol{D}} / \boldsymbol{t}_{V I B}=50$, the change in fluorescence anisotropy closely follows that observed in the limit of no excited state re-pumping. As $\boldsymbol{t}_{\boldsymbol{D}} / \boldsymbol{t}_{\boldsymbol{V} V \boldsymbol{B}}$ decreases, the anisotropy no longer rises with increasing $\boldsymbol{S}$ but reaches a plateau and then decreases. When $\boldsymbol{t}_{\boldsymbol{D}} / \boldsymbol{t}_{\boldsymbol{V} I \boldsymbol{B}}=10$, the maximum anisotropy change that can be induced is $0.29(\boldsymbol{S}=11.62)$. When $\boldsymbol{t}_{\boldsymbol{D}} / \boldsymbol{t}_{V I B}=1$, this reduces to approximately $0.047(\boldsymbol{S}=1.68)$. At lower values of $\boldsymbol{t}_{\boldsymbol{D}} / \boldsymbol{t}_{V I B}$, this form of the induced anisotropy is retained with a lower peak in $\Delta \boldsymbol{R}$ occurring at a lower value of $\boldsymbol{S}$ $\left(\boldsymbol{t}_{\boldsymbol{D}} / \boldsymbol{t}_{V I \boldsymbol{B}}=0.02 \Delta \boldsymbol{R}=3.29 \times 10^{-2} \& \mathrm{~S}=1.14\right)$ with $\Delta \boldsymbol{R}$ tending towards zero thereafter. With a population bottleneck in the lower level and in the absence of orientational relaxation, re-pumping of the excited state simply replaces the molecular transition dipole moment orientations that are initially removed as the dump pulse is applied. 
(a)

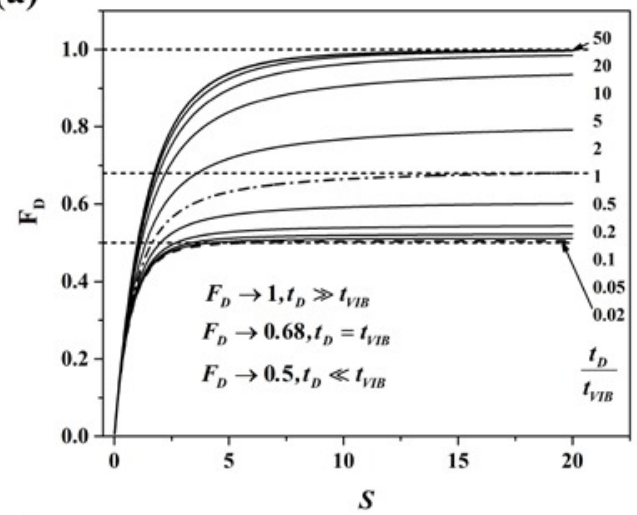

(c)

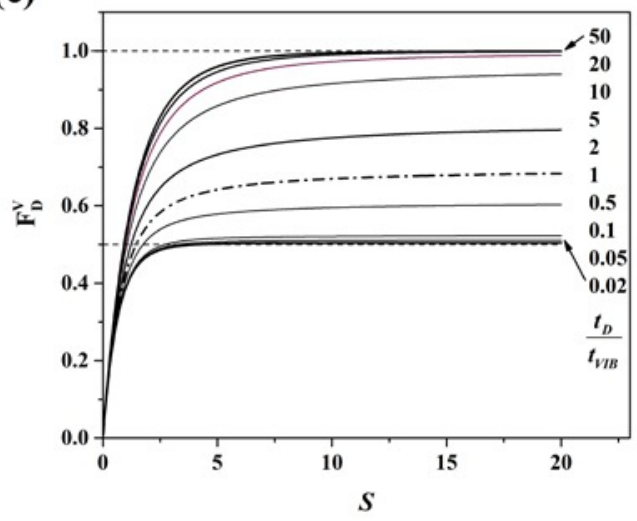

(b)

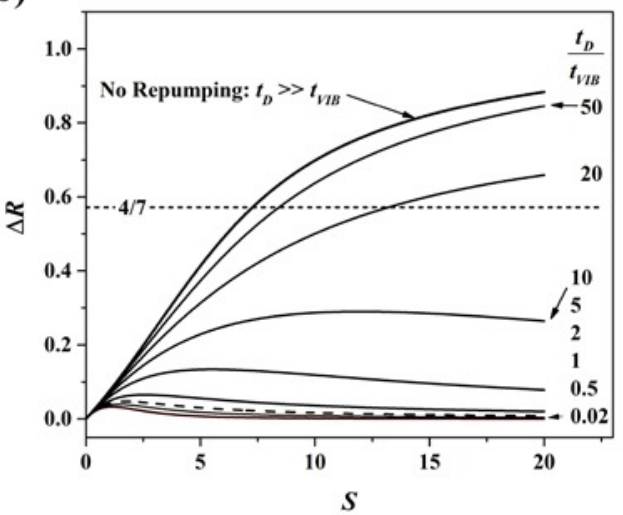

(d)

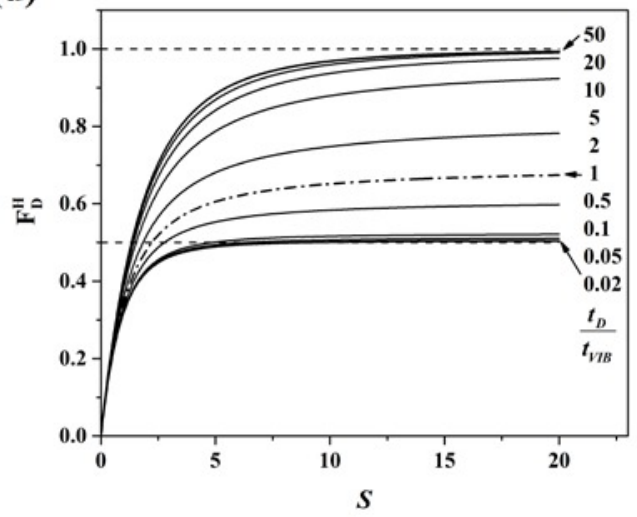

Figure 3: Simulation of $\boldsymbol{F}_{\boldsymbol{D}}, \boldsymbol{\Delta R}, \boldsymbol{F}_{\boldsymbol{D}}^{V}$ and $\boldsymbol{F}_{\boldsymbol{D}}^{\boldsymbol{H}}$ against dump energy $\left(\boldsymbol{S}=\sigma^{\text {STED }} \boldsymbol{E}_{\text {DUMP }} / \boldsymbol{A} \boldsymbol{h \boldsymbol { V } _ { D }}\right.$ ), for a population of twophoton excited molecules with an initial $\cos ^{4} \boldsymbol{\theta}$ orientational distribution $\left(\left\langle\boldsymbol{\alpha}_{20}(\Delta \mathbf{t}=\mathbf{0})\right\rangle=(4 / 7) \sqrt{ } 5,\left\langle\boldsymbol{\alpha}_{40}(\Delta \mathbf{t}=\mathbf{0})\right\rangle=8 / 21\right)$, as a function of $\boldsymbol{t}_{\boldsymbol{D}} / \boldsymbol{t}_{V I B}$ (the dump pulse width to ground state relaxation time ratio). From, T. A. Masters, N. Robinson, R. J. Marsh, D. A. Armoogum, T. S Blacker, B. Larijani, and A. J. Bain, J. Chem. Phys. 148, 134312 (2018): used in accordance with the Creative Commons Attribution (CC BY) license.

\subsection{Hexadecapolar alignment measurement by STED}

Simulations of the four STED observables with $\left\langle\alpha_{40}\right\rangle$ as the only variable parameter are shown in Figure 4. It can be seen (Figure 4(c)) that $\boldsymbol{F}_{\boldsymbol{D}}^{V}$ shows negligible $\left\langle\boldsymbol{\alpha}_{40}\right\rangle$ dependence at low and high values of $\boldsymbol{S}$ with a very slight variation in the turn over region. With increasing $\left\langle\boldsymbol{\alpha}_{40}\right\rangle$ in the high $\boldsymbol{S}$ limit, $\boldsymbol{F}_{\boldsymbol{D}}^{\boldsymbol{H}}$ and $\boldsymbol{F}_{\boldsymbol{D}}$ are reduced whilst the degree of STED depolarization $\boldsymbol{\Delta R}$ increases. The measurement of $\boldsymbol{F}_{\boldsymbol{D}}^{\boldsymbol{H}}$ has a considerably lower degree of experimental uncertainty than $\Delta \boldsymbol{R}^{11}$ and given the prior determination of $\boldsymbol{t}_{\boldsymbol{D}} / \boldsymbol{t}_{\boldsymbol{V} I \boldsymbol{B}}$, from $\boldsymbol{F}_{\boldsymbol{D}}^{V}$ and knowledge of $\boldsymbol{R}(\Delta t)$, analysis of $\boldsymbol{F}_{D}^{H}$ data should be the most accurate means of determining $\left\langle\alpha_{40}\right\rangle$. Measurement and analysis of $I_{\nu}(t)$ and $I_{H}(t)$ are also independent of any experimental g-factor arising from a polarization bias in fluorescence detection or (small) differences in the collection times of the two datasets. 
(a)

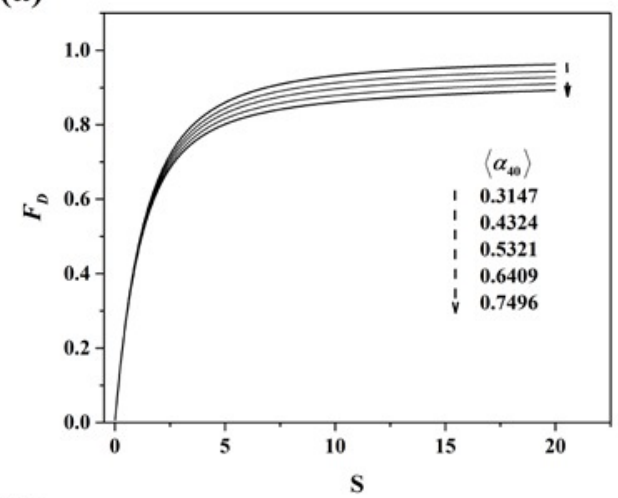

(c)

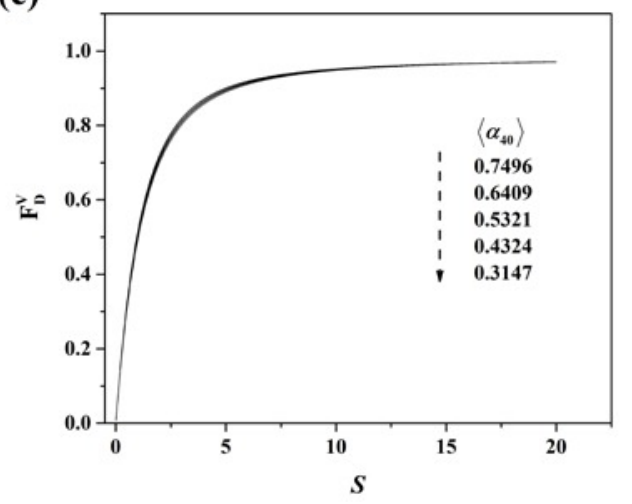

(b)

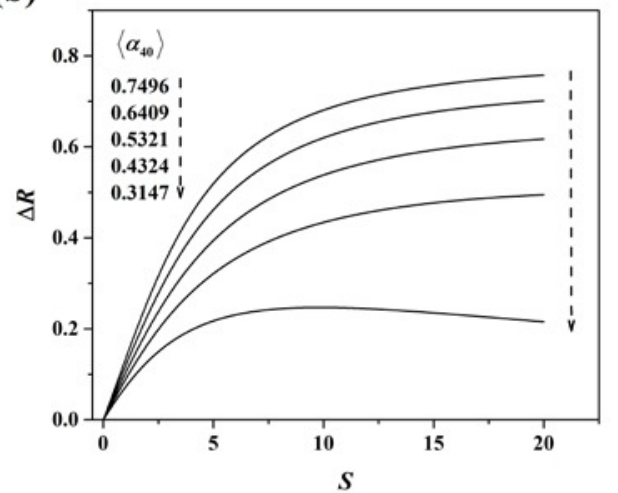

(d)

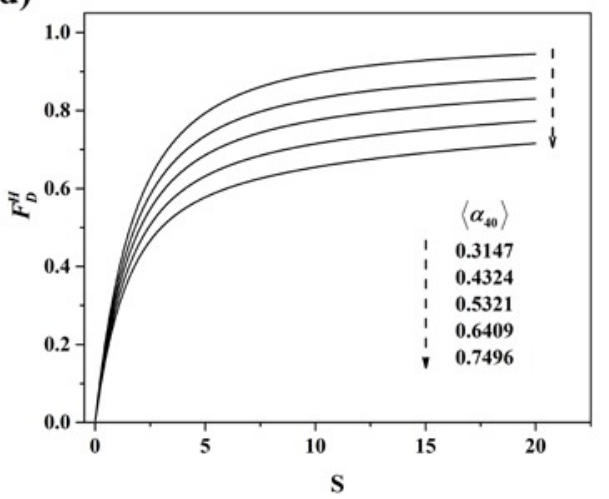

Figure 4: A simulation of the dependence of STED fluorescence observables on the degree of initial hexadecapolar $(K=4)$ alignment with $t_{D} / t_{V I B}=7$ and an initial quadrupolar alignment $\left\langle\alpha_{20}(\mathbf{0})\right\rangle / \sqrt{5}=0.54$. From, T. A. Masters, N. Robinson, R. J. Marsh, D. A. Armoogum, T. S Blacker, B. Larijani, and A. J. Bain, J. Chem. Phys. 148, 134312 (2018): used in accordance with the Creative Commons Attribution (CC BY) license.

\section{EXPERIMENTAL}

The experimental apparatus utilised to measure time resolved STED dynamics has been described in detail previously ${ }^{11}$. Two-photon excitation of a $40 \mu \mathrm{M}$ solution of recombinant EGFP in phosphate-buffered saline (PBS) at $800 \mathrm{~nm}$ was achieved using the partial output (55 nJ, 140 (FWHM) pulses at $250 \mathrm{kHz}$ ) of a regeneratively amplified Ti:Sapphire laser (Coherent Mira 900F, Coherent RegA 9000). Depletion pulses were derived from an optical parametric amplifier (OPA, Coherent 9400). The ca. 200 fs (FWHM) output pulses of the OPA (561-626 nm, maximum energy $300 \mathrm{~nJ}$ ) were temporally stretched using holographic grating pair (2400 grooves/mm, Optometrics LLC) to around $5 \mathrm{ps}$ in the determination of $\left\langle\alpha_{40}\right\rangle^{11}$.

\subsection{STED data analysis}

We model the depletion curves using Equations (16) to (19), inputting $\left\langle\boldsymbol{\alpha}_{20}(\Delta t)\right\rangle$ and $\left\langle\boldsymbol{\alpha}_{40}(\Delta t)\right\rangle$ which characterise

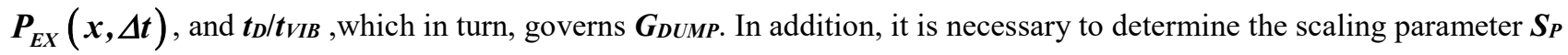


which connects the dump pulse energy $\left(\boldsymbol{E}_{\boldsymbol{D} U \boldsymbol{M} \boldsymbol{M})}\right)$ to the saturation parameter $\boldsymbol{S}^{11}$. The pre-dump orientational distribution at time $\Delta t$ following excitation can be calculated from,

$$
P_{E X}(x, \Delta t)=\frac{1}{2}\left[1+\frac{5}{2}[0.54] \exp \left(-\frac{\Delta t(n s)}{17.3}\right)\left(3 x^{2}-1\right)+\left\langle\alpha_{40}(\Delta t)\right\rangle \frac{3}{8}\left(35 x^{4}-30 x^{2}+3\right)\right]
$$

where 0.54 is the measured initial anisotropy in the absence of the dump pulse and $17.3 \mathrm{~ns}$ is the corresponding decay time. In the case of $F_{D}^{V}$ measurements, where the variation in $\left\langle\boldsymbol{\alpha}_{40}(\Delta t)\right\rangle$ makes a negligible contribution to the depletion curve, we can safely approximate the un-dumped orientational distribution by,

$$
P_{E X}(x, \Delta t)=\frac{1}{2}\left[1+\frac{5}{2}[0.54] \exp \left(-\frac{\Delta t(n s)}{17.3}\right)\left(3 x^{2}-1\right)+[0.381] \exp \left(-\frac{\Delta t(n s)}{0.3 \times 17.3}\right) \frac{3}{8}\left(35 x^{4}-30 x^{2}+3\right)\right]
$$

where 0.381 is the predicted value for $\left\langle\boldsymbol{\alpha}_{40}(\mathbf{0})\right\rangle$ based on the two-photon polarization measurements performed previously ${ }^{33}$. We also assume the relationship between $\tau_{40}$ and $\tau_{20}$ set out in Equation (8). In generating model curves to fit to $\boldsymbol{F}_{\boldsymbol{D}}^{\boldsymbol{H}},\left\langle\boldsymbol{\alpha}_{40}(\Delta t)\right\rangle$ becomes a fitting parameter in its own right,

$$
P_{E X}(x, \Delta t)=\frac{1}{2}\left[1+\frac{5}{2}[0.54] \exp \left(-\frac{\Delta t(n s)}{17.3}\right)\left(3 x^{2}-1\right)+\left\langle\alpha_{40}(\Delta t)\right\rangle \frac{3}{8}\left(35 x^{4}-30 x^{2}+3\right)\right]
$$

Model saturation curves were generated using Mathematica (Wolfram Research). For any given combination of $\Delta t, S_{P}$,

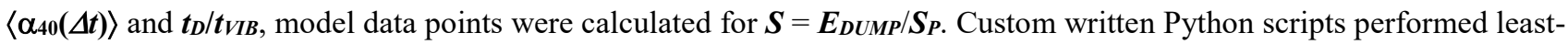
squares fitting of the model curves to the experimental data. The best fitting curves were judged as those that minimised the sum of the residuals weighted by the experimental uncertainties on the data points.

\section{RESULTS}

The hexadecapolar alignment measurements yielded values for $\left\langle\alpha_{40}\right\rangle$ for pump probe delay times spanning 200 ps to 3 ns, shown in Figure 5. The data can be fitted to a single exponential decay with a correlation time of $3.388 \mathrm{~ns}$. This is considerably shorter than that predicted for pure rotational diffusion $(5.19 \mathrm{~ns})$ and that obtained assuming the $\tau_{40}: \tau_{20}$ relationship (Equation (8)) holds in the presence of rotational diffusion and Homo-FRET. We can estimate the $\boldsymbol{K}=2$ and $\boldsymbol{K}=4$ homo-FRET rates from the difference between the measured and pure rotational diffusion rates,

$$
k_{H-F R E T}^{K}=\left(1 / \tau_{K 0}^{\text {Measured }}\right)-\left(1 / \tau_{K 0}^{\text {Rotation }}\right)
$$

This gives intrinsic Homo-FRET rates for $\boldsymbol{K}=2$ and $\boldsymbol{K}=4$ dipole alignment depolarization of $6( \pm 3) \times 10^{6} \mathrm{~s}^{-1}$ and $1.2( \pm 0.3)$ $\times 10^{8} \mathrm{~s}^{-1}$ respectively, a ratio of ca. 19:1 as opposed to 10/3:1 for pure rotational diffusion. The hexadecapolar transition dipole alignment appears considerably more sensitive to homo-FRET than the fluorescence anisotropy. The form of 
hexadecapolar dipole relaxation in the presence of homo-FRET has yet to be derived theoretically. With the assumption that, as in Equation (9), the overall correlation function can be described by the product of the correlation function for rotational diffusion together with that for energy migration ${ }^{30}$, the hexadecapolar dipole alignment dynamics would take the form,

$$
\left\langle\alpha_{40}(\Delta t)\right\rangle=\left\langle\alpha_{40}(\Delta t=0)\right\rangle \exp \left(-10 t / 3 \tau_{20}\right) \exp \left(-C \frac{R_{0}(K=4)^{3}}{375}\left(t / \tau_{F}\right)^{1 / 2}\right)
$$

where $\boldsymbol{R}_{\mathbf{0}}(\boldsymbol{K}=\mathbf{4})$ corresponds to the apparent Förster radius for hexadecapolar depolarization. A least squares fit to the data taking $\tau_{20}=19.5 \mathrm{~ns}$ and $\tau_{F}=2.75 \mathrm{~ns}$ yields an initial value for $\left\langle\boldsymbol{\alpha}_{40}(\mathbf{0})\right\rangle$ of $0.39( \pm 0.2)$ and a value for $\boldsymbol{R}_{\mathbf{0}}(\boldsymbol{K}=\mathbf{4})$ of $15( \pm 1) \mathrm{nm}$. The increase in the apparent Förster radius for homo-FRET over the value of $7 \mathrm{~nm}$ obtained above and in previous work ${ }^{30}$ for fluorescence depolarization is a necessary consequence arising from the need to account for the increased degree of homo-FRET depolarization in $\left\langle\boldsymbol{\alpha}_{40}(\Delta t)\right\rangle$ as is evident from Figure 5. Setting $\left\langle\boldsymbol{\alpha}_{40}(\mathbf{0})\right\rangle$ at 0.381 as we previously predicted for an emission dipole moment direction of $5.5^{\circ}$ relative to the transition tensor axis ${ }^{33}$ yields a similar value for $\boldsymbol{R}_{0}(\boldsymbol{K}=\mathbf{4})$ of $15.0( \pm 0.5) \mathrm{nm}$.

(a)

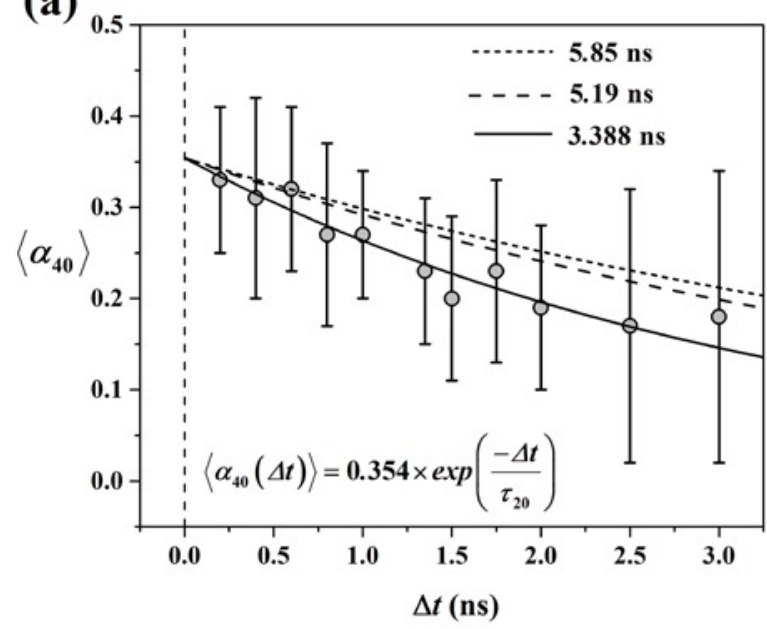

(b)

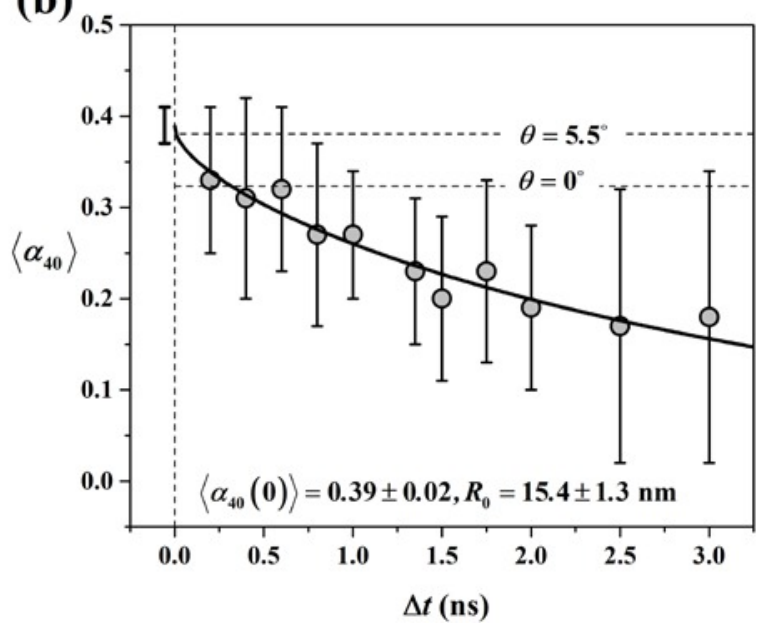

Figure 5 (a) Hexadecapolar transition dipole alignment as a function of pump-dump separation. The data can be fitted to a single exponential decay of $3.388 \mathrm{~ns}$, faster than the predicted decay for pure rotational diffusion (5.85 ns) and a single exponential decay of $5.19 \mathrm{~ns}$ assuming the relationship between $\tau_{40}$ and $\tau_{20}$ (Equation 8) holds in the presence of homoFRET. (b) A fit to the data using Equation (18) yields $\left\langle\boldsymbol{\alpha}_{40}(\mathbf{0})\right\rangle=0.39( \pm 0.02)$ and $\boldsymbol{R}_{\boldsymbol{0}}(\boldsymbol{K}=4)=15.5( \pm 1.3) \mathrm{nm}$. The vertical line indicates the uncertainty in $\left\langle\boldsymbol{\alpha}_{\mathbf{4 0}}(\mathbf{0})\right\rangle$. From: T. A. Masters, N. Robinson, R. J. Marsh, D. A. Armoogum, T. S Blacker B. Larijani, and A. J. Bain. J. Chem. Phys. 148, 134312 (2018): used in accordance with the Creative Commons Attribution (CC BY) license. 


\section{CONCLUSIONS}

The hexadecapolar transition dipole alignment dynamics, to the best of our knowledge, are the first measurement of higher order (rank $\boldsymbol{K}>2$ ) dipole moment correlations in resonance energy transfer. The increased sensitivity of the hexadecapolar alignment to depolarization over that reported by the fluorescence anisotropy is marked. Our results point to the perhaps not unexpected departure from the small step rotational diffusion relationship that exists between $\boldsymbol{K}=2$ and $\boldsymbol{K}=4$ dipole relaxation. We expect that the ability to probe higher order dipole alignment transfer in hetero-FRET would also provide a useful adjunct to current time resolved polarization techniques ${ }^{34,35}$ yielding new information and a clearer insight into resonance energy transfer dynamics.

\section{ACKNOWLEDGEMENTS}

We are grateful to the Engineering \& Physical Sciences Research Council for financial support of this work through PhD studentships awarded to TAM and TSB via the CoMPLEX doctoral training centre at UCL.

\section{REFERENCES}

[1] Huang, B., Bates, M. and Zhuang, X., "Super-resolution fluorescence microscopy.," Annu. Rev. Biochem. 78, 993-1016 (2009).

[2] Reisner, D. E., Field, R. W., Kinsey, J. L. and Dai, H., "Stimulated emission spectroscopy: A complete set of vibrational constants for $\tilde{X}^{1} A_{1}$ formaldehyde," J. Chem. Phys. 80(12), 5968-5978 (1984).

[3] Gryczynski, I., Kusba, J. and Lakowicz, J. R., "Light quenching of fluorescence using time-delayed laser pulses as observed by frequency-domain fluorometry," J. Phys. Chem. 98(36), 8886-8895 (1994).

[4] Haran, G., Morlino, E. A., Matthes, J., Callender, R. H. and Hochstrasser, R. M., "Femtosecond polarized pump- probe and stimulated emission spectroscopy of the isomerization reaction of rhodopsin," J. Phys. Chem. A 103(14), 2202-2207 (1999).

[5] Marsh, R., Armoogum, D. and Bain, A., "Stimulated emission depletion of two-photon excited states," Chem. Phys. Lett. 366(3-4), 398-405 (2002).

[6] Marsh, R. J., Leonczek, N. D., Armoogum, D. A., Monge, E. M. and Bain, A. J., "Stimulated emission depletion studies of molecular probe dynamics," Proc. SPIE 5925, Z. Gaburro and S. Cabrini, Eds., 59250C (2005).

[7] Armoogum, D. A., Marsh, R. J. and Bain, A. J., "Polarized stimulated emission depletion studies of two-photon excited states," Proc. SPIE 5222, Nanocrystals, Org. Hybrid Nanomater., 34-44 (2003).

[8] Bain, A. J., Marsh, R. J., Armoogum, D. A., Mongin, O., Porrès, L. and Blanchard-Desce, M., "Time-resolved stimulated emission depletion in two-photon excited states," Biochem. Soc. Trans. 31(5) (2003).

[9] Marsh, R. J., Leonczek, N. D., Armoogum, D. A., Porres, L., Mongin, O. M., Blanchard-Desce, M. H. and Bain, A. J., "Stimulated emission depletion dynamics in push-push polyenes," Proc. SPIE 5510, D. L. Andrews, G. Z. Cao, and Z. Gaburro, Eds., 117, Society of Photo-Optical Instrumentation Engineers (SPIE) (2004).

[10] Armoogum, D. A., Marsh, R. J., Nicolaou, N., Mongin, O., Blanchard-Desce, M. and Bain, A. J., "Stimulated emission depletion and fluorescence correlation spectroscopy of a branched quadrupolar chromophore," Proc. SPIE, 7030, Society of Photo-Optical Instrumentation Engineers (SPIE) (2008).

[11] Masters, T. A., Robinson, N. A., Marsh, R. J., Blacker, T. S., Armoogum, D. A., Larijani, B. and Bain, A. J., "Time-resolved Stimulated Emission Depletion and Energy Transfer Dynamics in Two-Photon Excited EGFP," J. Chem. Phys. 148, 134312 (2018).

[12] Bain, A. J. and McCaffery, A. J., "Multipolar evolution in two-and N-photon excitation," Chem. Phys. Lett. 108(3), 275-282 (1984).

[13] Bain, A. J. and McCaffery, A. J., "On the measurement of molecular anisotropies using laser techniques. III. Detection of the higher multipoles," J. Chem. Phys. 83(6), 2641-2645 (1985).

[14] Bain, A. J., "Multiphoton Processes," [Photonics], John Wiley \& Sons, Inc., Hoboken, NJ, USA, 279-320 (2015). 
[15] Bain, A. J., Chandna, P. and Bryant, J., "Picosecond polarized fluorescence studies of anisotropic fluid media. I. Theory,” J. Chem. Phys. 112(23), 10418 (2000).

[16] Bain, A. J., "Time-Resolved Polarised Fluorescence Studies of Ordered Molecular Systems," [An Introduction to Laser Spectroscopy], Springer US, Boston, MA, 171-210 (2002).

[17] Monge, E. M., Armoogum, D. A. and Bain, A. J., "Single- and two-photon time-resolved polarized fluorescence studies of probe molecule dynamics in nematic liquid crystals," Proc. SPIE 4797, Multiphot. Absorpt. Nonlinear Transm. Process. Mater. Theory, Appl., 264-274 (2003).

[18] Bain, A. J. and McCaffery, A. J., "On the measurement of molecular anisotropies using laser techniques. I. Polarized laser fluorescence," J. Chem. Phys. 83(6), 2627-2631 (1985).

[19] Debye, P. J. W., [Polar molecules], Chemical Catalog Company, Incorporated (1929).

[20] Bain, A. J., Chandna, P., Butcher, G. and Bryant, J., "Picosecond polarized fluorescence studies of anisotropic fluid media. II. Experimental studies of molecular order and motion in jet aligned rhodamine $6 \mathrm{G}$ and resorufin solutions," J. Chem. Phys. 112(23), 10435-10449 (2000).

[21] Fiorini, C., Charra, F. and Nunzi, J.-M., "Six-wave mixing probe of light-induced second-harmonic generation: example of dye solutions," J. Opt. Soc. Am. B 11(12), 2347 (1994).

[22] Lin, S., Hands, I. D., Andrews, D. and Meech, S. R., "Optically induced second harmonic generation by sixwave mixing: A novel probe of solute orientational dynamics," J. Phys. Chem. A 103(20), 3830-3836 (1999).

[23] Laage, D. and Hynes, J. T., “A Molecular Jump Mechanism of Water Reorientation,” Science (80-. ). 311(5762) (2006).

[24] Hochstrasser, R. M. and Negus, D. K., "Picosecond fluorescence decay of tryptophans in myoglobin.," Proc. Natl. Acad. Sci. U. S. A. 81, 4399-4403 (1984).

[25] Jovin, T. M., Lidke, D. S. and Post, J. N., "Dynamic and static fluorescence anisotropy in biological microscopy (rFLIM and emFRET)," J. Biomed. Opt. 5323, 1-12 (2004).

[26] Mattheyses, A. L., Hoppe, A. D. and Axelrod, D., "Polarized fluorescence resonance energy transfer microscopy.," Biophys. J. 87(4), 2787-2797 (2004).

[27] Anfinrud, P. A., Hart, D. E. and Struve, W. S., "Time-correlated photon-counting probe of singlet excitation transport and restricted rotation in Langmuir-Blodgett monolayers," J. Phys. Chem. 92(14), 4067-4073 (1988).

[28] Hart, D. E., Anfinrud, P. A. and Struve, W. S., "Excitation transport in solution: A quantitative comparison between GAF theory and time-resolved fluorescence profiles," J. Chem. Phys. 86(5), 2689-2696 (1987).

[29] Engstrom, S., Lindberg, M. and Johanssonb, L. B., "Fluorescence anisotropy of energy migration of rotating molecules in the presence," J. Chem. Phys. 96(10), 7528-7534 (1992).

[30] Clayton, A. H. A., Hanley, Q. S., Arndt-Jovin, D. J., Subramaniam, V. and Jovin, T. M., "Dynamic Fluorescence Anisotropy Imaging Microscopy inthe Frequency Domain (rFLIM),” Biophys. J. 83(3), 1631-1649 (2002).

[31] Fredrickson, G. H., "Concentration Depolarization of Fluorescence in the Presence of Molecular Rotation," J. Chem. Phys. 88(9), 5291-5299 (1988).

[32] Patterson, G. H., Piston, D. W. and Barisas, B. G., "Förster distances between green fluorescent protein pairs.," Anal. Biochem. 284(2), 438-440 (2000).

[33] Masters, T. A., Marsh, R. J., Blacker, T. S., Armoogum, D. A., Larijani, B. and Bain, A. J., "Polarized twophoton photoselection in EGFP: Theory and experiment," J. Chem. Phys. 148, 134311 (2018).

[34] Masters, T. A., Marsh, R. J., Armoogum, D. A., Nicolaou, N., Larijani, B. B. and Bain, A. J., "Restricted State Selection in Fluorescent Protein Forster Resonance Energy Transfer," J. Am. Chem. Soc. 135(21), 7883-7890 (2013).

[35] Blacker, T. S., Chen, W. Y., Avezov, E., Marsh, R. J., Duchen, M. R., Kaminski, C. F. and Bain, A. J., "Investigating state restriction in fluorescent protein FRET using time-resolved fluorescence and anisotropy," J. Phys. Chem. C 121(3), 1507-1514 (2017).

[36] Siegman, A. E., [Lasers], University Science Books (1986). 